\title{
Anabolic effects of a $G$ protein-coupled receptor kinase inhibitor expressed in osteoblasts
}

\author{
Robert F. Spurney, Patrick J. Flannery, Sanford C. Garner, Krairerk Athirakul, \\ Shiguang Liu, Farshid Guilak, and L. Darryl Quarles
}

Division of Nephrology, Department of Medicine, Duke University and Durham Veterans Administration Medical Centers, Durham, North Carolina, USA

Address correspondence to: Robert F. Spurney, Box 3014,

Duke University Medical Center, Durham, North Carolina 27710, USA.

Phone: (919) 660-6869; Fax: (919) 684-4476; E-mail: spurn002@mc.duke.edu.

Received for publication November 19, 2001, and accepted in revised form April 9, 2002.

\begin{abstract}
G protein-coupled receptors (GPCRs) play a key role in regulating bone remodeling. Whether GPCRs exert anabolic or catabolic osseous effects may be determined by the rate of receptor desensitization in osteoblasts. Receptor desensitization is largely mediated by direct phosphorylation of GPCR proteins by a family of enzymes termed GPCR kinases (GRKs). We have selectively manipulated GRK activity in osteoblasts in vitro and in vivo by overexpressing a GRK inhibitor. We found that expression of a GRK inhibitor enhanced parathyroid hormone (PTH)/PTH-related peptide (PTHrP) receptor-stimulated cAMP generation and inhibited agonist-induced phosphorylation of this receptor in cell culture systems, consistent with attenuation of receptor desensitization. To determine the effect of GRK inhibition on bone formation in vivo, we targeted the expression of a GRK inhibitor to mature osteoblasts using the mouse osteocalcin gene 2 (OG2) promoter. Transgenic mice demonstrated enhanced bone remodeling as well as enhanced urinary excretion of the osteoclastic activity marker dexoypyridinoline. Both osteoprotegrin and OPG ligand mRNA levels were altered in calvaria of transgenic mice in a pattern that would promote osteoclast activation. The predominant effect of the transgene, however, was anabolic, as evidenced by an increase in bone density and trabecular bone volume in the transgenic mice compared with nontransgenic littermate controls.
\end{abstract}

J. Clin. Invest. 109:1361-1371 (2002). doi:10.1172/JCI200214663.

\section{Introduction}

Osteoporosis is a common medical problem that leads to the loss of the structural integrity of bone, resulting in substantial morbidity and mortality (1). In the United States alone, about 1.5 million osteoporosis-related bone fractures occur each year. Antiresorptive therapies are commonly used as treatment and prevent bone loss by inhibiting osteoclast-mediated bone resorption (1). Restoration of the trabecular networking and structural integrity of the skeleton by selective stimulation of bone-forming osteoblasts has proven more challenging. Several G protein-coupled receptor (GPCR) systems in osteoblasts appear promising for promoting osteogenesis (2), including parathyroid hormone (PTH), E- and F-series prostaglandins, and possibly a cation-sensing receptor (2-7). Unfortunately, pharmacologic strategies that target GPCRs have been limited by coupling of osteoclast-mediated bone resorption with osteoblast-mediated bone formation $(2,8,9)$. Indeed, GPCR-stimulation of osteoblast-mediated bone formation is typically followed by varying degrees of osteoclast-mediated bone resorption, which mitigates the anabolic effects of GPCRs.

Clinical trials with PTH, however, suggest that the kinetics of receptor activation/deactivation may determine whether GPCR stimulation is catabolic or anabolic.
In this regard, intermittent treatment with low-dose PTH stimulates new bone formation in excess of bone resorption $(4,6,7)$, whereas continuous administration of PTH causes bone loss $(8,9)$. Thus, desensitization of the PTH/PTH-related peptide (PTHrP) receptor might explain the differing effects of intermittent compared with continuous administration of PTH on bone. In this scenario, the continuous presence of agonist rapidly attenuates the responsiveness of most GPCRs; in contrast, the intermittent stimulation of the receptor permits prolonged activation of signaling pathways leading to net bone formation. The availability of molecular strategies to modulate GPCR desensitization permits the assessment of the molecular mechanism underlying the anabolic effects of GPCRs in bone.

Desensitization of GPCRs is largely mediated by direct phosphorylation of receptor serine and threonine residues by a family of kinases termed GPCR kinases or GRKs (10-13). GRKs are a family of enzymes containing six members (11-13). Because of the highly restricted tissue distribution of GRK1 and GRK4 $(11,12)$, either GRK2, GRK3, GRK5, or GRK6 are likely to be the important regulators of GPCR responsiveness in bone. In this regard, recent studies from this laboratory and by others suggest that GRK2 and/or GRK3 play dominant roles in regulating responsiveness of GPCR systems in bone 
(14-16). Developing strategies for modulating GRK2 and GRK3 activity might therefore provide a mechanism for regulating GPCR responsiveness. Because currently there are no specific chemical GRK inhibitors, investigators have developed alternative methods for modulating GRK function. One such strategy takes advantage of the observation that domains in the C terminus of GRK2 and GRK3 are critical for targeting of the enzymes to activated receptors at the cell membrane (17-20). The C termini of GRK2 and GRK3 are enzymatically inactive but can compete with GRKs for receptor substrate (17-20). Overexpressing C-terminal domains of either GRK2 (GRK2-CT) or GRK3 (GRK3-CT) therefore inhibits the ability of GRKs to bind receptor substrate, thus preventing agonist-induced phosphorylation of GPCRs and, in turn, enhancing GPCR signaling. This strategy for inhibiting activity of GRKs has been used successfully both in cell culture systems as well as in whole animal models (17-20).

To begin investigating the role of GRKs in bone formation, we first studied the effect of GRK inhibition on GPCR signaling in cell culture models using the $\mathrm{PTH} / \mathrm{PTHrP}$ receptor as a model system. We found that expression of the GRK2-CT enhanced PTH/PTHrP receptor signaling in HEK293 cells transfected with the $\mathrm{PTH} / \mathrm{PTHrP}$ receptor cDNA as well as in the rat osteosarcoma cell line ROS 17/2.8 cells, which express an endogenous receptor for PTH/PTHrP. This increase in $\mathrm{PTH} / \mathrm{PTHrP}$ receptor responsiveness was associated with a reduction in agonist-induced phosphorylation of the PTH/PTHrP receptor. To study the role of GRKs in vivo, we created transgenic mice that expressed the GRK2-CT in osteoblasts using a $1.3-\mathrm{kb}$ fragment of the mouse osteocalcin gene 2 (OG2) promoter (21) to target expression of the GRK inhibitor to mature osteoblasts (22). Transgenic mice demonstrated an increase in both osteoblastic and osteoclastic activity as well as alterations in osteoprotegrin (OPG) and OPG ligand (OPGL) mRNA levels in mouse calvaria, which had a pattern that would tend to promote osteoclast activation. Although both bone formation and bone resorption were enhanced in transgenic mice, the net effect of the transgene was anabolic, as evidenced by an increase in bone density and trabecular bone volume in the transgenic animals compared with nontransgenic littermate controls. Taken together, these data indicate that GRKs regulate GPCR systems in bone and suggest that pharmacological manipulation of GRK activity in bone may be a strategy for promoting osteogenesis.

\section{Methods}

Materials. Rat PTH1-34 and human [125I]-(Nle8,18, $\left.\mathrm{Tyr}^{34}\right)$ PTH1-34amide were obtained from Sigma-Aldrich (St. Louis, Missouri, USA) and Amersham International (Amersham, United Kingdom), respectively. Pfu DNA polymerase was obtained from Stratagene (La Jolla, California, USA) and Taq DNA polymerase was obtained from Promega Corp. (Madison, Wisconsin, USA). All PCR primers were prepared by Operon Technologies Inc.
(Alameda, California, USA). Cell culture reagents were obtained from Life Technologies Inc. (Gaithersburg, Maryland, USA), and $\left[{ }^{3} \mathrm{H}\right]$-adenine was purchased from New England Nuclear (Boston, Massachusetts, USA). Human embryonic kidney (HEK293) cells were obtained from American Type Culture Collection (Rockville, Maryland, USA). L. Darryl Quarles provided the rat osteosarcoma cell line ROS 17/2.8 cells (23). The E86 ecotropic retroviral packing cells were provided by Clay Smith (Duke University Medical Center) (24). The 12CA5 Ab was purchased from Roche Molecular Biochemicals (Indianapolis, Indiana, USA). Rabbit polyclonal Ab's to the C terminus of GRK2 (25) were a kind gift of Robert J. Lefkowitz (Duke University Medical Center). The cDNA for the GRK2-CT was also a gift of Robert J. Lefkowitz and was produced as described previously (18-20). A 1.3-kb fragment of the OG2 promoter was provided by Gerard Karsenty (Baylor College of Medicine, Houston, Texas, USA) (21).

Isolation of a $c D N A$ encoding the rat $P T H / P T H r P$ receptor. A cDNA clone encoding the rat $\mathrm{PTH} / \mathrm{PTHrP}$ receptor (26) was isolated by RT-PCR using the high-fidelity DNA polymerase $P f u$ and total RNA prepared from the rat osteosarcoma cell line ROS 17/2.8 cells as described previously (14). The 12CA5-tagged PTH/PTHrP receptor was produced as previously described (14).

Creation of a retroviral GRK2-CT. The pLuv retroviral vector was provided by Clay Smith (27). To generate the GRK2-CT retroviral vector, we used PCR and the highfidelity DNA polymerase Pfu (Stratagene) to insert BspHI and BamHI sites immediately $5^{\prime}$ and $3^{\prime}$ to the start and stop codons, respectively. PCR was performed with the bovine GRK2 cDNA (14) as the template and using the forward primer GACACAAAAGGAATCATGATACTGGACAGTGACCAG and the reverse primer TGGGCCATGGCGGCCGCGCTTCTGCAGGTCGACTCTAG. For the PCR reaction, the thermocycler was set at $94^{\circ} \mathrm{C}$ for 30 seconds, $50^{\circ} \mathrm{C}$ for 30 seconds, and $72^{\circ} \mathrm{C}$ for $2 \mathrm{~min}$ utes. The PCR product was cut with $\mathrm{BspHI} / \mathrm{BamHI}$ and was inserted into the unique $\mathrm{NcoI} / \mathrm{BamHI}$ restriction sites in the pLuv vector to create pLuv-GRK2-CT. Sequencing of the constructs using the dideoxy method (28) confirmed that the construct was inserted in the proper orientation and in-frame.

Development of ecotropic retroviral producer cell lines. The E86 ecotropic retroviral packing cell line was a gift of Clay Smith (24). To create cells stably expressing the pLuv-GRK2-CT vector, cells were transfected with $5 \mu \mathrm{g}$ pLuv-GRK2-CT and $1 \mu \mathrm{g}$ of pSV2-neo (American Type Culture Collection) vector containing a neomycin-resistant cassette. After transfection, cells were maintained in medium containing $700 \mu \mathrm{g} / \mathrm{ml} \mathrm{G} 418$. For serum containing retroviral supernatant, cells were washed with DMEM and then grown for 2 days in DMEM supplemented with $10 \%$ heat-inactivated FCS. The supernatant was then collected and centrifuged to remove cell debris. All supernatant aliquots were stored at $-70^{\circ} \mathrm{C}(27)$.

Culture and transfection of HEK293 cells. HEK293 cells were grown and subcultured as described previously (14). 
For transfection, HEK293 cells were plated in either 60$\mathrm{mm}$ dishes or six-well plastic culture dishes $\left(9.5 \mathrm{~cm}^{2} /\right.$ well $)$ (Corning-Costar Corp., Cambridge, Massachusetts, USA) and grown to approximately $80 \%$ confluence. Cells were then transfected using the calcium phosphate method (28). To express PTH/PTHrP receptors, we used $1 \mu \mathrm{g}$ of plasmid DNA per milliliter of transfection solution. Preliminary experiments suggested that these amounts of plasmid DNA optimized the level of receptor expression and transfection efficiency $(-20-40 \%)$. For each transfection, $1 \mathrm{ml}$ of transfection solution was added (in drops) to a single $60-\mathrm{mm}$ dish or to two wells of a six-well plastic culture dish $\left(9.5 \mathrm{~cm}^{2} /\right.$ well $)$ (Corning-Costar Corp.). In the cotransfection experiments, the GRK-CT plasmid DNA was included in the transfection solution at a concentration of $5 \mu \mathrm{g} / \mathrm{ml}$. Following an overnight incubation, the DNA solution was replaced with DMEM supplemented with $10 \%$ heat-inactivated FCS, penicillin (100 $\mathrm{U} / \mathrm{ml})$, and streptomycin $(100 \mu \mathrm{g} / \mathrm{ml})$. HEK293 cells were studied after 48 hours following transfection.

Culture and infection of ROS 17/2.8 cells. ROS 17/2.8 cells were grown and subcultured as described previously (23). For retroviral infection, cells were plated the night before at a density of $2 \times 10^{4}$ to $4 \times 10^{4}$ cells $/ \mathrm{ml}$ in six-well plastic culture dishes $(9.5$ $\mathrm{cm}^{2} /$ well) (Corning-Costar Corp.). The following day, $1 \mathrm{ml}$ of freshly thawed retroviral vector supernatant containing $10 \mu \mathrm{g} / \mathrm{ml}$ polybrene (Sigma-Aldrich) was added to each well. After 1 hour, an additional milliliter of medium was added. Medium was changed the following day, and cells were studied after 2 days following infection. Preliminary studies using a pLuv vector designed to express green fluorescent protein indicated that this protocol resulted in an $80-90 \%$ infection rate (data not shown).

Radioligand-binding studies. Radioligand-binding studies were performed using cell monolayers as described previously (14). Equilibrium-binding data were analyzed by the method of Scatchard (29) to give estimates of the maximal number of specific binding sites $\left(B_{\max }\right)$ and apparent equilibrium $K_{\mathrm{d}}$ by fitting the data to a nonlinear model using the ENZFITTER computer program (Elsevier-Biosoft, Cambridge, United Kingdom). Data are expressed as femtomoles per million cells.

Measurement of cAMP generation. Generation of cAMP was measured by the method of Salomon et al. (30) as described previously (14). Data are expressed as the percentage of increase above basal cAMP generation.

Immunoblotting of GRKs. Expression GRK2, GRK3, and the GRK2-CT were evaluated with a rabbit polyclonal $\mathrm{Ab}(25)$ that recognizes the C termini of both GRKs using techniques described previously (14).

Immunoprecipitation of epitope-tagged PTH/PTHrP receptors and immunoblotting of immunoprecipitates. HEK293 cells were treated with the indicated concentrations of agonist or vehicle, then immunoprecipitation of PTH/PTHrP receptors was performed as described previously (14). Proteins were separated on $9 \%$ polyacrylamide gel with $0.1 \%$ SDS as described by
Laemmli (31). After drying the gels, phosphorylated proteins were detected by autoradiography.

To determine the amount of PTH/PTHrP receptor protein in the immunoprecipitates a portion of the immunoprecipitated proteins were separated using $9 \%$ polyacrylamide gel with $0.1 \%$ SDS and were transferred from the polyacrylamide gel to nitrocellulose (Bio-Rad Laboratories Inc., Richmond, California, USA) using a Milliblot semidry transfer apparatus (Millipore Corp., Bedford, Massachusetts, USA), according to the directions of the manufacturer. The nitrocellulose was blocked for 1 hour in BLOTTO. After blocking, the 12CA5 Ab was added at a concentration of $0.5 \mu \mathrm{g} / \mathrm{ml}$ in BLOTTO. The blot was incubated at room temperature for 1 hour with gentle rocking followed by three washes with T-TBS. The horseradish peroxidase-labeled secondary Ab (Amersham International) was added at a dilution of 1:2,000 in BLOTTO. After rocking for 1 hour at room temperature, the blot was washed once with $0.2 \%$ Tween $20-\mathrm{TBS}$ (TTBS) and twice with TBS. Proteins were detected by enhanced chemiluminescence (ECL) according to the manufacturer's specifications (Amersham International).

Transgene construction and creation of transgenic mice expressing the GRK2-CT. The transgene was constructed in the vector pcDNA 3.1 (Invitrogen Corp., San Diego, California, USA). The mouse OG2 promoter was provided by Gerard Karsenty (21). The GRK2-CT was provided by Robert Lefkowitz and contains amino acids 495-689 of bovine GRK2 ligated to the human $\beta$-globulin polyadenylation signal $(18,32)$. To create the transgene a $1.3-\mathrm{kb}$ fragment of the mouse OG2 promoter was ligated into the unique KpnI/EcoRI site of pcDNA 3.1 vector. The GRK2-CT, including the human $\beta$-globulin polyadenylation signal, was ligated in the unique EcoRI/XbaI site of pcDNA 3.1 just $3^{\prime}$ to the promoter sequence.

Transfection of ROS 17/2.8 with the GRK2-CT transgene. ROS 17/2.8 cells were grown and subcultured as described previously (23). To determine if our transgene could be expressed by osteoblastic cell lines, we created a construct in which transgene expression was driven solely by the OG2 promoter by removing the CMV promoter sequences in pcDNA 3.1 using the restriction enzymes MluI and HindIII. After filling in the overhangs with Pfu DNA polymerase, the construct was circularized with T4 DNA ligase. To transfect ROS 17/2.8 cells, cells were plated in 60-mm dishes (Corning-Costar Corp.) and grown to approximately $80 \%$ confluence. Cells were then transfected with the Transfast system (Promega Corp.) according to the directions of the manufacturer, using $5 \mu \mathrm{g}$ of plasmid DNA. After selection in G418 $(700 \mu \mathrm{g} / \mathrm{ml}$ concentration) for 2 weeks, cells were harvested, and evaluation of transgene expression was evaluated by immunoblotting as described above.

Creation of transgenic mice expressing the GRK2-CT. To create transgenic mice, the transgene was purified by cesium chloride centrifugation (28) and then linearized by cutting with the restriction enzymes HindIII/XbaI. The linearized transgene was then separated from 
vector sequences on a $0.8 \%$ agarose gel and extracted from the gel using the QIAquick Gel Extraction Kit (QIAGEN Inc., Valencia, California, USA). To remove endotoxins, the transgene was further purified by treatment with the EndoFree Kit (QIAGEN Inc.), according to the directions of the manufacturer. The purified transgene was injected into the pronuclei of one-cell mouse embryos and then surgically implanted into pseudopregnant females in the transgenic facility at Duke University Medical Center. Transgenic mice were identified by the PCR as described below. All animal procedures were approved by the Animal Care and Use Committee of Duke University Medical Center.

Screening for transgenic mice by the PCR. To screen for our transgenic animals we performed PCR using Taq DNA polymerase (Promega Corp.) and 100-200 ng of DNA prepared from mouse tails using the DNeasy Tissue Kit (QIAGEN Inc.) according to the directions of the manufacturer. The PCR reaction was performed for 40 cycles using the primer pairs encompassing nucleotides 1567-1588 (GGAATCAAGCTACTGGACAGTG) of the bovine GRK2 cDNA (33) and nucleotides 469-450 (GAAATTGGACAGCAAGAAAG) in the $3^{\prime}$ untranslated region of the human $\beta$-globulin mRNA (32) with the thermal cycle set at $94^{\circ} \mathrm{C}$ for 30 seconds, $50^{\circ} \mathrm{C}$ for 30 seconds, and $72^{\circ} \mathrm{C}$ for 2 minutes.

Transgene $m R N A$ expression. To investigate tissue specific expression of the transgene, we reverse transcribed total cellular RNA from mouse tissues and then performed PCR (RT-PCR). The reverse-transcription reaction was performed with Superscript reverse transcriptase (Life Technologies Inc.) and oligo-dT primers using $2 \mu \mathrm{g}$ of total cellular RNA prepared with the Trizol reagent (Life Technologies Inc.) according to the directions of the manufacturer. Prior to the PCR reaction, total cellular RNA was treated with DNAase (Life Technologies Inc.) according to the directions of the manufacturer. The reverse transcription reaction was performed using Superscript reverse transcriptase (Life Technologies Inc.) and oligo-dT primers according to the directions of the manufacturer. PCR was performed for 30 cycles using Taq DNA polymerase (Promega Corp.) and primer pairs encompassing nucleotides 1567-1588 (GGAATCAAGCTACTGGACAGTG) of the bovine GRK2 cDNA (33) and nucleotides 469-450 (GAAATTGGACAGCAAGAAAG) in the $3^{\prime}$ untranslated region of the human $\beta$-globulin mRNA (32), with the thermal cycler set at $94^{\circ} \mathrm{C}$ for 30 seconds, $50^{\circ} \mathrm{C}$ for 30 seconds, and $72^{\circ} \mathrm{C}$ for 2 minutes. Control PCR reactions were performed for 30 cycles using GAPDH primers (CLONTECH Laboratories Inc.) with the thermal cycler set at $94^{\circ} \mathrm{C}$ for 30 seconds, $58^{\circ} \mathrm{C}$ for 30 seconds, and $72^{\circ} \mathrm{C}$ for 2 minutes. PCR products were separated on $1 \%$ agarose gels and visualized by staining with ethidium bromide.

Measurement of cAMP generation by mouse calvaria. To determine if the GRK2-CT transgene affected GPCR responsiveness in our transgenic animals, we measured PTH-induced cAMP generation by mouse calvaria ex vivo. For the experiments, a symmetrical fragment of mouse calvarium containing portions of the occipital, frontal, and parietal bones was isolated aseptically from 6-weekold mice. Calvaria were cut into right and left halves by dividing the bony fragment along the sagital suture line. Right and left halves were each divided into three fragments of equal size. Preliminary experiments suggested that this procedure for obtaining portions of mouse calvaria resulted in bony fragments that varied by less than $15 \%$ by weight. Individual bony fragments of calvaria were placed in separate wells of a six-well plastic culture dish $\left(9.5 \mathrm{~cm}^{2} /\right.$ well) (Corning-Costar Corp.). For the cAMP measurements, the bony fragments were covered with DMEM containing $1 \% \mathrm{FCS}$ and $2 \mu \mathrm{Ci} / \mathrm{ml}\left[{ }^{3} \mathrm{H}\right]$-adenine (New England Nuclear). After 90 minutes, $100 \mu \mathrm{M}$ IBMX was added to the medium. Twenty minutes later, calvaria were stimulated with $100 \mathrm{nM}$ PTH1-34. For the initial studies, calvaria were incubated with either vehicle (Dulbecco's-PBS [D-PBS]) or PTH1-34 for 10 minutes, and data points are the results of triplicate measurements from four separate experiments. In the time-course studies, calvaria were stimulated with vehicle (D-PBS) or PTH1-34 for either 5 minutes or 10 minutes, and data points are the results of duplicate measurements from six separate experiments. Following incubation with PTH134 , the medium was aspirated and the reaction stopped by adding STOP solution (2.5\% perchloric acid containing $100 \mu \mathrm{M}$ cAMP and $1 \mu \mathrm{Ci}\left[{ }^{14} \mathrm{C}\right]$-cAMP) and placing the samples on ice. The samples were then processed as described above for measurement of cAMP generation.

Expression of OPG and OPGL $m R N A$. To investigate the effect of the transgene on OPG and OPGL mRNA levels in bone, we performed semiquantitative RT-PCR using 2 $\mu \mathrm{g}$ of total cellular RNA isolated from mouse calvaria. The reverse transcription reaction was performed with Superscript reverse transcriptase (Life Technologies Inc.) and oligo-dT primers using RNA prepared with the Trizol reagent (Life Technologies Inc.) according to the directions of the manufacturer. PCR for OPG was performed for 25 cycles using Taq DNA polymerase (Promega Corp.) and primers previously shown to specifically amplify OPG (34) with the thermal cycle set at $94^{\circ} \mathrm{C}$ for 30 seconds, $50^{\circ} \mathrm{C}$ for 30 seconds, and $72^{\circ} \mathrm{C}$ for $90 \mathrm{sec}-$ onds. PCR for OPGL was performed for 25 cycles using Taq DNA polymerase (Promega Corp.) and primers previously shown to specifically amplify OPGL (35) with the thermal cycler set at $94^{\circ} \mathrm{C}$ for 30 seconds, $52^{\circ} \mathrm{C}$ for $30 \mathrm{sec}$ onds, and $72^{\circ} \mathrm{C}$ for 2 minutes. Preliminary studies indicated that the midpoint of the linear phase of template amplification occurred after 25 cycles of PCR in nontransgenic control mice. Control PCR reactions were performed for 25 cycles with GAPDH primers (CLONTECH Laboratories Inc.) with the thermal cycler set at $94^{\circ} \mathrm{C}$ for 30 seconds, $58^{\circ} \mathrm{C}$ for 30 seconds, and $72^{\circ} \mathrm{C}$ for 2 minutes. PCR products were separated on $1 \%$ agarose gels and visualized by staining with ethidium bromide.

Bone histomorphometry. Quantitative histomorphometric analysis of trabecular bone in tibias of 6-week-old mice was performed using techniques described previously (36). Mice were given an intraperitoneal injection of 

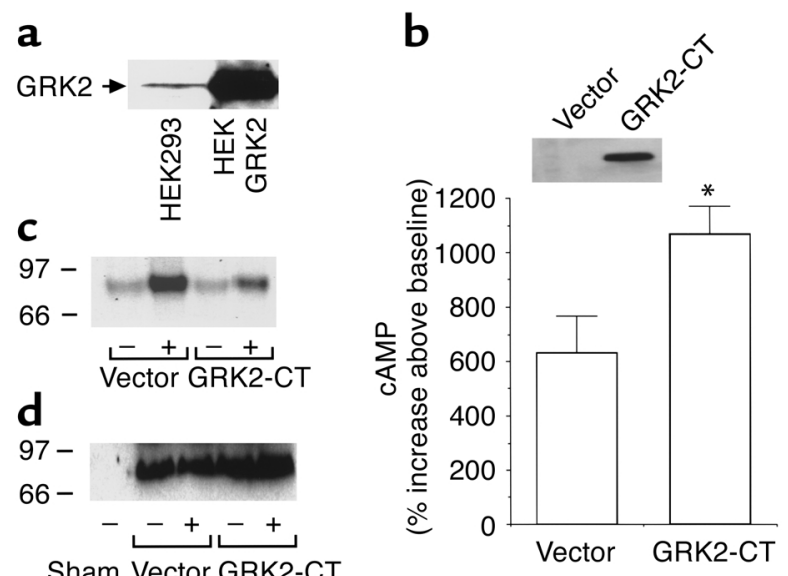

\begin{abstract}
Figure 1
Expression of the GRK2-CT enhances PTH/PTHrP receptor responsiveness and inhibits agonist-induced phosphorylation of the PTH/PTHrP receptor. HEK293 cells were cotransfected with the rat PTH/PTHrP receptor cDNA and either the GRK2-CT or empty vector. Two days after transfection, expression of GRK2 and the GRK2-CT was assessed by immunoblotting as described in Methods using an Ab that recognizes the $C$ terminus of both GRK2 and GRK3 (25). In parallel experiments, we investigated the effect of the GRK2-CT on PTH/PTHrP receptor responsiveness by measuring CAMP generation and agonist-induced phosphorylation of the PTH/PTHrP receptor by immunoprecipitation of 12CA5-tagged PTH/PTHrP receptors, as described in Methods. Apparent molecular mass is indicated in kilodaltons. (a) In lane 1, HEK293 cells express predominantly GRK2. A slightly smaller protein corresponding to the GRK3 was detected by immunoblotting only after prolonged exposure of the radiographic film. Lane 2 is a positive control prepared from HEK293 cells transfected with the GRK2 cDNA. (b) Cotransfection of the GRK2-CT significantly enhanced PTH/PTHrP receptor responsiveness compared with cells cotransfected with empty vector. The inset shows that the increase in PTH/PTHrP receptor responsiveness was associated with high levels of GRK2-CT expression in this model system. (c) Cotransfection of the GRK2-CT inhibited agonist-induced phosphorylation of the PTH/PTHrP receptor. (d) The amount of PTH/PTHrP receptor in the immunoprecipitates was assessed by immunoblotting using the 12CA5 Ab. Similar amounts of PTH/PTHrP receptor were immunoprecipitated from cells cotransfected with either the GRK2-CT or empty vector. ${ }^{*} P<0.05$ vs. vector.
\end{abstract}

tetracycline $\mathrm{HCl}(30 \mu \mathrm{g} / \mathrm{g}$ body weight) followed by an injection of calcein $(15 \mu \mathrm{g} / \mathrm{g}$ body weight $)$ on days 3 and 8 before sacrifice, respectively. After harvesting, nondecalcified sections of the right tibia were fixed in $70 \%$ ethanol, followed by staining with Villanueva stain, embedding in methyl methacrylate, and sectioning longitudinally at a thickness of 5-10 $\mu \mathrm{m}$. The 5- $\mu \mathrm{m}$ sections were stained with Goldner's stain and analyzed under transmitted light, and the 10- $\mu \mathrm{m}$ Villanueva prestained sections were analyzed under fluorescent light as described previously (36) using the Osteomeasure digitizing system (Osteometrics Inc., Atlanta, Georgia, USA). To analyze the effect of the transgene on cortical bone volume, the left tibia was prepared as described above for light microscopy and sectioned transversely at the tibulofibular junction to standardize the location of the transverse section. Fivemicrometer sections were stained with Goldner's stain, and bone area (percentage of tissue area) was measured under transmitted light as described previously (36). The terminology and units used are those recommended by the histomorphometry nomenclature committee of the American Society of Bone and Mineral Research (37).

Bone densitometry and dry ash bone weight. Bone mineral density (BMD; given in grams per square centimeter) of femurs was assessed at 6 weeks of age using a LUNARPIXI $\mathrm{MUS}_{\text {b }}$ bone densitometer (Lunar Corp., Madison, Wisconsin, USA). The instrument was calibrated before scanning sessions using a phantom with known BMD according to the manufacturer's guidelines. Dry ash bone weight was measured as described previously (36).

Measurement of urinary dexoypyridinoline excretion. Mice were placed in metabolic cages, and urine was collected for 24 hours. The urine volume was measured prior to storage at $-70^{\circ} \mathrm{C}$. Dexoypyridinoline (DPD) excretion was quantitated using the Pyrilinks-D assay kit (Metra Biosystems Inc., Mountain View, California, USA). Data were expressed as picomoles of DPD per 24 hours.

Statistical analysis. Data are presented as the mean plus or minus SEM. For comparisons between two groups, statistical significance was assessed using a Student $t$ test.

\section{Results}

A GRK inhibitor enhances PTH/PTHrP receptor signaling in cell culture systems. To investigate the role of GRKs in regulating GPCR responsiveness in bone, we initially evaluated the effects of a dominant negative GRK construct (the GRK2-CT) (17-20) using the PTH/PTHrP receptor as our model system. For the experiments, we cotransfected HEK293 cells with the rat PTH/PTHrP receptor and the GRK2-CT. As shown in Figure 1a, HEK293 cells express predominantly GRK2 as seen by immunoblotting using an $\mathrm{Ab}$ that recognizes the $\mathrm{C}$ termini of both GRK2 and GRK3 (25). Figure $1 \mathrm{~b}$ shows that coexpression of the rat PTH/PTHrP receptor and the GRK2-CT significantly enhanced PTH/PTHrP receptor responsiveness compared with cells coexpressing the PTH/PTHrP receptor and empty vector. The increase in $\mathrm{PTH} / \mathrm{PTHrP}$ receptor responsiveness was associated with high levels of GRK2CT expression in this model system (Figure 1b, inset). As shown in Table 1, expression of the GRK2-CT had no effect on either binding affinity or cell surface expression of the PTH/PTHrP receptor. These data suggest that inhibition of endogenous GRK activity enhances PTH/PTHrP receptor responsiveness in HEK293 cells.

To determine if inhibition of endogenous GRK activity attenuates agonist-specific phosphorylation of GPCRs, we investigated the effect of the GRK2-CT on $\mathrm{PTH}$-induced phosphorylation $\mathrm{PTH} / \mathrm{PTHrP}$ receptor using a 12CA5-tagged construct that we have used previously to monitor agonist-induced phosphorylation of the PTH/PTHrP receptor (14). For the experiments, HEK293 cells were cotransfected with a 12CA5-tagged rat PTH/PTHrP receptor and either the GRK2-CT or empty vector. Two days after transfection, cells were loaded with ${ }^{32} \mathrm{P}$ and then stimulated with PTH1-34 or vehicle. After 10 minutes, cells were harvested and 
Table 1

Binding of [ $\left.{ }^{125} \mathrm{I}\right] \mathrm{PTH}$ to cells cotransfected with empty vector or the GRK2-CT

\begin{tabular}{lcc}
\hline & $B_{\max }(\mathrm{fmol} /$ million cells $)$ & $K_{\mathrm{d}}(\mathrm{nM})$ \\
Empty vector & $186 \pm 18$ & $1.7 \pm 0.4$ \\
GRK2-CT & $166 \pm 12$ & $1.6 \pm 0.8$
\end{tabular}

Results of three assays. Data were analyzed by the method of Scatchard (29) to obtain the $B_{\max }$ and equilibrium $K_{\mathrm{d}}$. Data are presented as the mean $\pm \mathrm{SEM}$.

immunoprecipitation was performed as described in Methods. As shown in Figure 1c, stimulation of transfected HEK293 cells with 100 nM PTH1-34 caused phosphorylation of a broad band of $\sim 80 \mathrm{kDa}$, which previously we have demonstrated is the $\mathrm{PTH} / \mathrm{PTHrP}$ receptor (14). Phosphorylation of the $\mathrm{PTH} / \mathrm{PTHrP}$ receptor protein was attenuated in cells cotransfected with the GRK2-CT compared with cells cotransfected with empty vector. Figure $1 \mathrm{~d}$ shows that similar amounts of $\mathrm{PTH} / \mathrm{PTHrP}$ receptor were immunoprecipitated in cells cotransfected with either empty vector or the GRK2-CT. These data indicate that inhibition of GRK activity in our HEK293 cell system attenuates agonist-induced phosphorylation of the PTH/PTHrP receptor.

We next investigated the effect of GRK inhibition on $\mathrm{PTH} / \mathrm{PTHrP}$ receptor responsiveness in the rat osteosarcoma cell line ROS 17/2.8 cells, which express an endogenous receptor for $\mathrm{PTH} / \mathrm{PTHrP}(26)$. For the experiments, we first determined if ROS 17/2.8 cells express GRK2 and/or GRK3 by immunoblotting using an $\mathrm{Ab}$ that recognizes both GRKs (25). As shown in Figure 2a, ROS 17/2.8 cells express GRK2 and lesser levels of GRK3. To express the GRK2-CT in ROS 17/2.8 cells, we used a retroviral system that results in high levels of protein expression in $80-90 \%$ of the cells (data not shown). Figure $2 \mathrm{~b}$ shows that infection of ROS 17/2.8 cells with the GRK2-CT significantly enhanced PTH/PTHrP receptor responsiveness compared with cells infected with empty vector. The increase in PTH/PTHrP receptor responsiveness was associated with high levels of GRK2-CT expression in this model system (Figure 2c). These data, taken together with previous studies (38), suggest that inhibition of endogenous GRK activity in an osteoblast cell line enhances GPCR responsiveness.

Creation of a GRK2-CT transgene. To determine if modulation of GRK activity affects bone formation in vivo, we used a $1.3-\mathrm{kb}$ fragment of the mouse OG2 promoter (see Figure 3a) to target expression of the GRK2-CT to mature osteoblasts (22). Before generating transgenic mice, we determined if our transgene was expressed in the rat osteosarcoma cell line ROS 17/2.8 cells. The GRK2-CT construct was designed so that expression of the transgene was driven solely by the OG2 promoter (see Methods). For the studies, ROS 17/2.8 cells were transfected with either empty vector or the GRK2-CT transgenic construct. The mammalian expression vector used in the experiments contained a neomycinresistant cassette that permitted G418 selection. After selecting stable transfectants in G418, expression of the GRK2-CT was investigated by immunoblotting. As shown in Figure 3b, the GRK2-CT was expressed by ROS 17/2.8 cells. These data are consistent with the notion that our GRK2-CT transgene is targeted for expression in mature osteoblasts.

Effect of GRK2-CT transgene on osteoblast-mediated bone formation in vivo. To determine if our transgene affected bone formation in vivo, the linearized construct was injected into the pronuclei of one-cell mouse embryos and then surgically reimplanted into pseudopregnant females. Mice were screened for incorporation of the transgene by PCR, using primers specific for the transgene (see Figure 3a). Two independent lines were established from the founder animals and identical results were obtained using the progeny from these independent founder lines. We next determined tissue-specific expression of our transgene by reverse transcription of total cellular RNA isolated from mouse tissues, followed by PCR using transgenespecific primers (Figure 3a). As shown in Figure 3c, a PCR product of the appropriate size was detected in bone from transgenic mice using GRK2-CT primers when an RT reaction was performed prior to PCR. A small amount of GRK2-CT PCR product was also detected in the brain, as has been reported by other investigators using this promoter sequence (39). Sequencing of the PCR product confirmed the presence of transgene mRNA. No GRK2-CT PCR products were detected in other tissues from the transgenic mice or in any of the tissues from the nontransgenic littermate controls (Figure 3d). The control PCR reaction (GAPDH) confirmed that the RT reaction was successful in the tissues examined. These data a

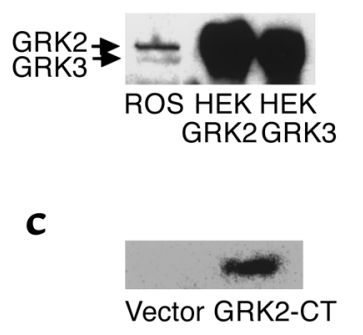

b

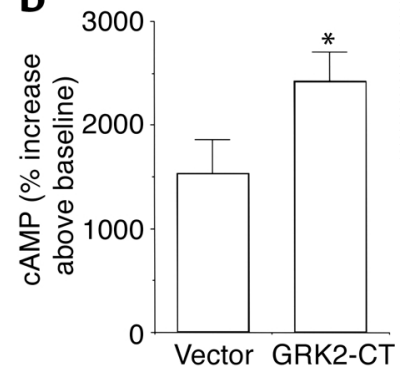

\section{Figure 2}

Expression of the GRK2-CT enhances PTH/PTHrP receptor responsiveness in cells that express an endogenous PTH/PTHrP receptor. The GRK2-CT was expressed in ROS $17 / 2.8$ cells using a retroviral system as described in Methods. Two days after infection, expression of GRK2, GRK3, and the GRK2-CT was assessed by immunoblotting as described in Methods, using an $A b$ that recognizes the $C$ terminus of both GRK2 and GRK3 (25). In parallel experiments, cAMP generation was measured to determine the effect of the GRK2-CT on PTH/PTHrP receptor responsiveness. (a) In lane 1, ROS 17/2.8 cells express GRK2 and lesser amounts of GRK3. Lane 2 and lane 3 are positive controls prepared from HEK293 cells transfected with either the GRK2 cDNA (lane 2) or the GRK3 cDNA (lane 3). (b) The GRK2-CT significantly enhanced PTH/PTHrP receptor responsiveness compared with cells infected with empty vector. (c) The increase in PTH/PTHrP receptor responsiveness was associated with high levels of GRK2-CT expression in this model system. ${ }^{*} P<0.05$ vs. vector. 
suggest that transgene mRNA expression is targeted to bone with low levels of ectopic expression in brain.

We next determined if expression of the transgene was associated with enhanced responsiveness of GPCRs in bone by measuring PTH-induced cAMP generation ex vivo using mouse calvaria. We found that PTH-induced cAMP generation was significantly increased in calvaria from transgenic mice compared with nontransgenic littermate controls 10 minutes after the addition of PTH1-34 (transgenic, $147 \% \pm 29 \%$ increase vs. nontransgenic, $99 \% \pm 16 \%$ increase above baseline; $P<0.05)$. Additional experiments were performed to determine if the tempo of PTH-induced cAMP generation was altered by the transgene. We found that cAMP generation was similar 5 minutes $(105 \% \pm 27 \%$ increase above baseline) and 10 minutes $(91 \% \pm 25 \%$ increase above baseline $)$ after the addition of PTH1-34 in nontransgenic mice. In contrast, PTH-induced cAMP generation increased significantly between the 5- and 10-minute time points in the transgenic animals $(124 \% \pm 22 \%$ vs. $168 \% \pm 10 \%$ increase above baseline; $P<0.05)$. As in the initial studies, PTH-induced cAMP generation was also increased in transgenic mice compared with the nontransgenic littermate controls at the 10 -minute time point $(P<0.025)$. $\mathrm{PTH} / \mathrm{PTHr} \mathrm{P}$ receptor responsiveness tended to be increased in transgenic mice compared with the control animals at the 5-minute time point, but this difference was not statistically significant. Taken together, these data are consistent with the notion that inhibition of endogenous GRK activity in osteoblasts enhances GPCR responsiveness in bone.

To investigate the phenotype of our transgenic mice, we first performed BMD measurements on femurs isolated from mice at 6 weeks of age. Data were similar in male and female animals; we therefore combined these data for the statistical analysis. There was a statistically significant increase in BMD in transgenic animals compared with nontransgenic littermate controls (wild-type, $54.2 \pm 2.1 \mathrm{mg} / \mathrm{cm}^{2}$, vs. transgenic, $59.0 \pm 1.2 \mathrm{mg} / \mathrm{cm}^{2}$; $P<0.05)$. Similarly, dry ash bone weight tended to be increased in transgenic mice compared with nontransgenic littermates; however, this difference did not reach statistical significance (wild-type, $14.1 \pm 2.9$ $\mathrm{mg}$, vs. transgenic, $17.5 \pm 1.3 \mathrm{mg}$; $P=\mathrm{NS})$. These data suggest that expression of the transgene increases bone mineral content.

We next performed bone histomorphometry on tibias isolated from

\section{Figure 3}

mice at 6 weeks of age. Data were similar in male and female animals and were therefore combined for the statistical analysis. As shown in Figure 4 and Table 2, trabecular bone volume was increased in transgenic mice compared with nontransgenic littermate controls. This increase in trabecular bone volume was caused by an increase in trabecular thickness without an increase in the number of trabeculae. In the transgenic animals, osteoid surface, osteoid thickness, and osteoblast number were significantly increased compared with control animals. Osteoblast surface, erosive surface, and osteoclast number tended to be increased in the transgenic animals, but these differences did not reach statistical significance. The increase in osteoid volume and osteoid thickness reflects accelerated bone formation since the dynamic indices of bone formation showed an increase in both the mineral apposition rate as well as the bone formation rate (see Figure 4 and Table 2), and the mineralization lag time was not different between transgenic mice and nontransgenic littermate controls. Cortical bone cross-sectional area was not different in transgenic
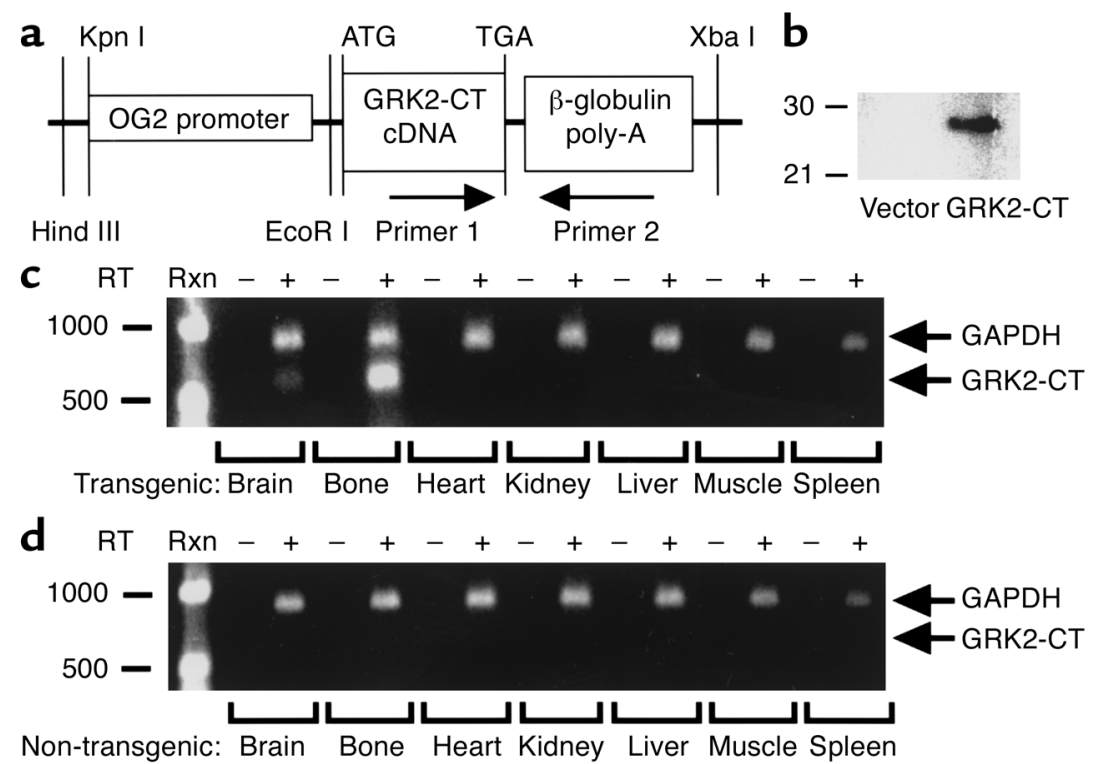

Transgene construction and expression in ROS $17 / 2.8$ cells as well as in mouse tissues. (a) The GRK2-CT transgene containing the 1.3-kb mouse OG2 promoter, the GRK2-CT, and the human $\beta$-globulin polyadenylation signal. Also shown are the approximate locations of the PCR primers (primer 1 and primer 2). (b) ROS 17/2.8 cells were transfected with a mammalian expression vector containing our transgene and a neomycin-resistant cassette. The constructs was designed so that GRK2-CT expression was driven solely by the OG2 promoter (see Methods). Following G418 selection, GRK2-CT expression was investigated by immunoblotting. The GRK2-CT was expressed by ROS $17 / 2.8$ cells transfected with the vector containing our transgene, but not in cells transfected with empty vector. Apparent molecular mass is indicated in kDa. (c and d) PCR or RT-PCR was performed using total cellular RNA prepared from the indicated mouse tissues as described in Methods. Molecular size is indicated in base pairs. (c) A PCR product of the appropriate size was detected in bone from transgenic mice when an RT reaction was performed prior to PCR. A small amount of GRK2-CT PCR product was also detected in the brain, as has been reported by other investigators (40). (d) No GRK2-CT PCR products were detected in tissues from nontransgenic littermate controls. Control PCR reactions revealed a PCR product of the appropriate size in all tissues using the GAPDH primers when an $\mathrm{RT}$ reaction was performed prior to PCR. 

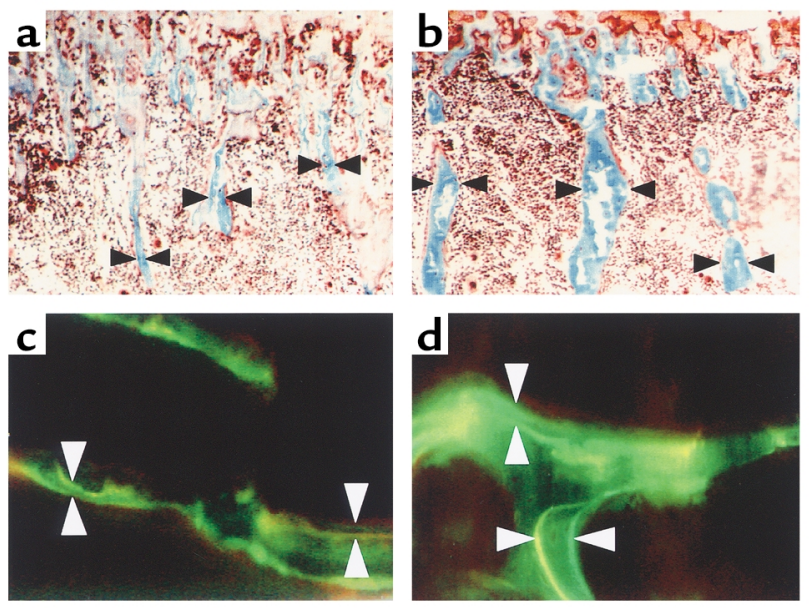

\section{Figure 4}

Bone histomorphology in transgenic mice and in nontransgenic littermate controls. Mice were given an intraperitoneal injection of tetracycline $\mathrm{HCl}$ followed by an injection of calcein on days 3 and 8 prior to sacrifice, respectively. After harvesting, nondecalcified sections of tibias were prepared for histomorphologic analysis as described in Methods. (a and $\mathbf{b}$ ) Representative 5- $\mu \mathrm{m}$ sections of trabecular bone visualized under transmitted light from nontransgenic controls and transgenic mice, respectively. ( $\mathbf{c}$ and $\mathbf{d}$ ) Representative $10-\mu \mathrm{m}$ sections of trabecular bone visualized under fluorescent light from control animals and transgenic mice, respectively. Transgenic mice demonstrated an increase in trabecular thickness compared with nontransgenic littermate controls as indicated by the distance between the black arrowheads in $\mathbf{a}$ and $\mathbf{b}$. Under fluorescent light, there was an increase in the distance between the fluorescent labels in transgenic mice compared with control animals as indicated by the distance between the white arrowheads in $\mathbf{c}$ and $\mathbf{d}$.

mice compared with nontransgenic littermate controls (wild-type, $74.5 \% \pm 0.5 \%$, vs. transgenic, $75.5 \% \pm 2.5 \%$; $P=$ NS). Taken together, these data are consistent with the notion that inhibition of GRK activity in mature osteoblasts caused an increase in trabecular bone volume by enhancing osteoblast-mediated bone formation.

Effect of the transgene on DPD excretion and expression of $O P G$ and OPGL $m R N A$. To investigate the effect of the transgene on osteoclast-mediated bone resorption in vivo, we measured urinary excretion of DPD, which is a marker of both osteoclast number and activity (40). As shown in Figure 5a, urinary excretion of DPD was enhanced in transgenic mice compared with nontransgenic littermate controls. These data suggest that targeting expression of the transgene to mature osteoblasts was accompanied by enhanced osteoclastic activity and, in turn, increased bone resorption.

The osteoclast inhibitory factor OPG and the osteoclast differentiation factor OPGL are potent regulators of osteoclast development (41). Both OPG and OPGL are produced by osteoblasts (41), and agonists of several GPCR systems have been shown to modulate their production $(35,42,43)$. We therefore determined if our transgene affected OPG and OPGL mRNA levels. For the experiments, we performed semiquantitative RT-PCR on total cellular RNA isolated from calvaria of transgenic mice and nontransgenic littermate controls at 6 weeks of age. As shown in Table 3 and in Figure 5, b-d, OPG mRNA levels were decreased in transgenic animals compared with nontransgenic littermate controls. In contrast,

Table 2
OPGL mRNA levels were increased compared with the controls. These data suggest that the transgene has divergent effects on OPG and OPGL mRNA levels in bones from transgenic animals.

\section{Discussion}

GPCR agonists are important endogenous regulators of bone remodeling (44-49) and are potentially useful as pharmacological agents for regenerating the trabecular architecture of bone (2, 4-9). Treatment with GPCR agonists, however, is often limited by catabolic effects due to coupling of osteoblast-mediated bone formation to osteoclast-mediated resorption $(2,8,9)$. The observations that intermittent PTH administration has potent anabolic actions and continuous infusion has catabolic effects on bone $(8,9)$ raises the possibility that GPCR desensitization is an important determinant of skeletal homeostasis. In the current investigations, we directly establish a relationship between GPCR desensitization and osteogenic potential by inhibiting GPCR desensitization in

Effect of the GRK-CT on trabecular bone at 6 weeks of age

\begin{tabular}{|c|c|c|c|}
\hline & Controls & Transgenic & $P$ value \\
\hline \multicolumn{4}{|l|}{ atic indices of bone formation: } \\
\hline abecular volume (BV/TV) (\%) & $8.24 \pm 1.63$ & $12.66 \pm 1.57$ & $P<0.05$ \\
\hline becular width (Tb.Th) $(\mu \mathrm{m})$ & $26.3 \pm 1.12$ & $30.5 \pm 1.76$ & $P<0.05$ \\
\hline becular number (Tb.N) (per mm) & $2.14 \pm 0.20$ & $2.25 \pm 0.11$ & NS \\
\hline eoid surface/bone surface (OS/BS) (\%) & $11.8 \pm 1.91$ & $19.1 \pm 2.94$ & $P<0.05$ \\
\hline teoblast surface/bone surface (Ob.S/BS) (\%) & $11.1 \pm 1.83$ & $16.2 \pm 2.77$ & NS \\
\hline sive surface/bone surface (ES/BS) (\%) & $11.9 \pm 2.04$ & $13.2 \pm 2.00$ & NS \\
\hline mber of osteoblast/total area (N.Ob/T.A) (per mm²) & $38.9 \pm 5.35$ & $72.5 \pm 12.5$ & $P<0.005$ \\
\hline umber of osteoclasts/total area (N.Oc/T.A) (per $\left.\mathrm{mm}^{2}\right)$ & $18.2 \pm 4.66$ & $24.1 \pm 4.21$ & NS \\
\hline steoid thickness (O.Th.) $(\mu \mathrm{m})$ & $2.88 \pm 0.53$ & $4.26 \pm 0.55$ & $P<0.05$ \\
\hline \multicolumn{4}{|l|}{ ynamic indices of bone formation: } \\
\hline neral apposition rate (MAR) $(\mu \mathrm{m} /$ day $)$ & $2.05 \pm 0.31$ & $3.39 \pm 0.49$ & $P<0.025$ \\
\hline ne formation rate $(\mathrm{BFR} / \mathrm{BS})\left(\mu \mathrm{m}^{3} / \mu \mathrm{m}^{2} /\right.$ day $)$ & $0.40 \pm 0.09$ & $0.89 \pm 0.09$ & $P<0.005$ \\
\hline ineralizing perimeter (MS/BS) (\%) & $19.3 \pm 3.85$ & $27.2 \pm 3.38$ & NS \\
\hline ineralization lag time (Mlt) (days) & $0.84 \pm 0.27$ & $0.89 \pm 0.15$ & NS \\
\hline
\end{tabular}

Values are the mean \pm SEM. Five nontransgenic littermate controls (three males and two females) and five transgenic mice (three males and two females) were studied. 

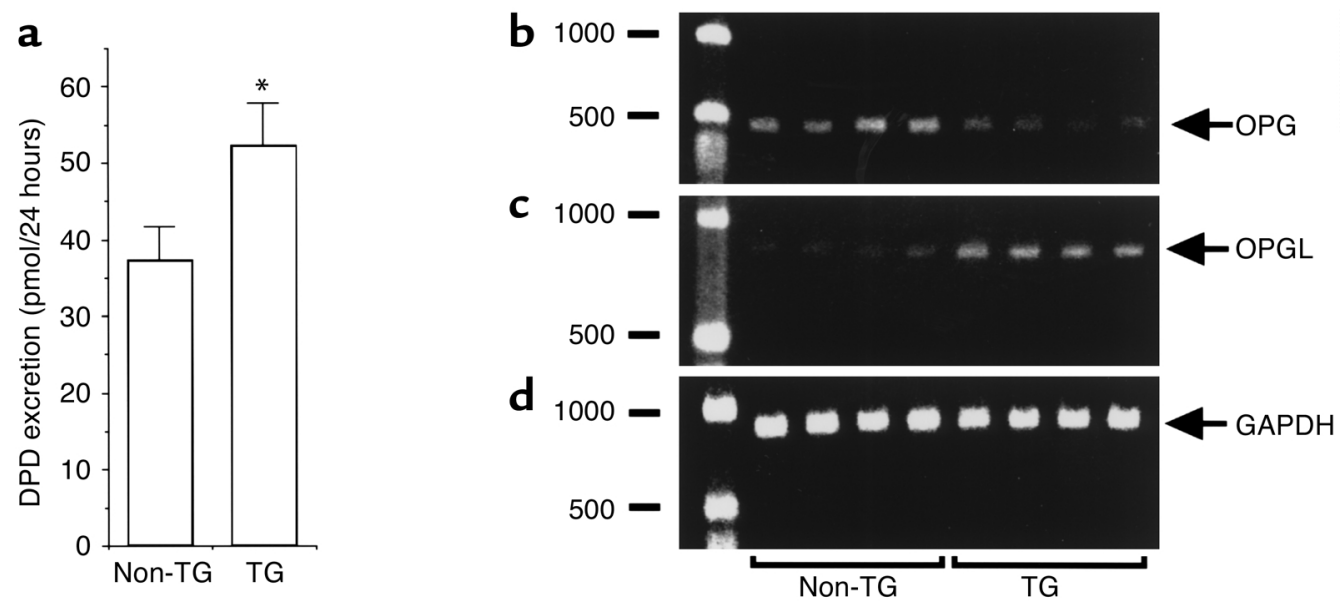

Figure 5

Effect of the transgene on urinary excretion of DPD and expression OPG and OPGL mRNA in mouse calvaria. (a) Excretion of DPD in urine was measured as an index of osteoclast-mediated bone resorption in vivo (40). Excretion of urinary DPD was significantly enhanced in transgenic (TG) mice compared with nontransgenic (Non-TG) littermate controls. (b-d) Total cellular RNA was prepared from mouse calvaria prior to performing semiquantitative RT-PCR as described in Methods. Molecular size is indicated in base pairs. (b) The OPG PCR product was decreased in transgenic mice compared with nontransgenic littermate controls. (c) The OPGL PCR product was increased in transgenic animals compared with control animals. The GAPDH control PCR reaction shown in c confirmed that the RT reaction was successful in the animals studied. ${ }^{*} P<0.025$ vs. nontransgenic littermate controls.

osteoblasts with a dominant negative C-terminal fragment of GRK2 (18-20). In HEK293 cells transfected with the PTH receptor cDNA, as well as in osteoblasts expressing an endogenous PTH receptor, we found that GRK2-CT overexpression enhanced PTH-induced cAMP generation (Figure 1 and Figure 2). Enhanced PTH receptor signaling also was associated with a reduction in agonist-induced phosphorylation of the PTH receptor in HEK293 cells cotransfected with the PTH receptor and the GRK2-CT (Figure 1). Our findings differ from those of Malecz et al. (16), which failed to inhibit agonist-specific phosphorylation of the $\mathrm{PTH} / \mathrm{PTHrP}$ receptor responsiveness by transient transfection of GRK2-CT into cells stably expressing the PTH/PTHrP receptor. The different results may be due to the higher levels of CRK2-CT expression attained with the retroviral system $(24,27)$ compared with the relatively inefficient expression using transient transfection strategies. Regardless, our findings that GRK2-CT produced a statistically significant increase in PTH-induced cAMP generation in ROS 17/2.8 osteoblasts, together with our previous observations (14) and the findings of other investigators $(15,16,38)$, provide compelling evidence that GRKs contribute to regulating PTH receptor responsiveness.

Based on the in vitro findings, we predicted that the targeted overexpression of the GRK2-CT to osteoblasts would inhibit desensitization of endogenous GPCRs and, in turn, prolong the actions of endogenous GPCR ligands leading to stimulation of bone remodeling. Indeed, we found that PTH-induced cAMP generation by mouse calvaria was enhanced ex vivo in transgenic mice compared with nontransgenic littermate controls. Moreover, targeted expression of a GRK

Table 3 inhibitor to mature osteoblasts using the OG2 promoter enhanced osteoblast-mediated bone formation (Table 2). Since mature osteoblasts were the primary target for transgene expression and these cells contain several GPCRs $(2,50)$, the effects on bone remodeling are likely due to the persistent activity of endogenous osteoblastic GPCRs. This receptor "overactivity" resulted in an increase in osteoblast-mediated deposition of extracellular matrix and mineral apposition at individual bone remodeling sites (Table 2). We also observed an increase in the osteoid-covered bone surfaces as well as osteoid thickness in transgenic mice that is likely due to accelerated bone formation since the mineralization lag time (Mlt) was not changed (Table 2), indicating that the hyperosteoidosis was not due to defective mineralization. Lastly, accelerated bone formation was associated with an increase in osteoblast number, which could result from either enhanced osteoblast proliferation, increased recruitment of new osteoblasts, or prolongation of osteoblast life span. We favor the latter possibility since the OG2 promoter drives expression of the GRK inhibitor in postmitotic mature osteoblasts (22), and GRK inhibition has antiproliferative effects on osteoblast cell lines (ref. 51 and unpublished data). Interestingly, recent studies by Jilka et al. (52) suggest that GPCR agonists such as PTH are

Effect of the GRK2-CT on OPG and OPGL mRNA levels

\begin{tabular}{lrl}
\hline & Nontransgenic & \multicolumn{1}{c}{ Transgenic } \\
OPG (optical density ratio OPG/GAPDH) & $0.29 \pm 0.041$ & $0.11 \pm 0.033^{\mathrm{A}}$ \\
OPGL (optical density ratio OPGL/GAPDH) & $0.082 \pm 0.016$ & $0.14 \pm 0.013^{\mathrm{B}}$ \\
\hline
\end{tabular}

Values are the mean \pm SEM. Five mice were studied in each group. ${ }^{A} P<0.01$ or ${ }^{B} P<0.025$ vs. nontransgenic littermate control. 
potent inhibitors of osteoblast apoptosis both in vitro and in vivo, thereby prolonging osteoblast life span. Moreover, the present studies as well as studies by other investigators (38) indicate that GRK inhibition enhances GPCR responsiveness to agonists such as PTH in osteoblast cell lines. While further studies will be necessary to determine the role of apoptosis in promoting the increase in osteoblast numbers observed in the present studies, it is tempting to speculate that the increase in the number of mature osteoblasts in our transgenic mice may have been mediated by inhibiting osteoblast apoptosis.

Normally, stimulation of osteoblast-mediated bone formation is linked to osteoclast-mediated bone resorption. This effect is due, at least in part, to the balance between the osteoclast inhibitory factor OPG and the osteoclast differentiation factor OPGL $(35,42,43)$, which are released from osteoblast-precursors and mature osteoblasts (41). By bone histomorphometry, which can detect only major changes in bone resorption, we failed to observe evidence for increased osteoclast-mediated bone resorption in OG2-GRK-CT transgenic mice. However, the increase in DPD excretion in the transgenic mice suggest enhanced osteoclastic activity. These data prompted us to exam the effect of our transgene on OPG and OPGL mRNA levels in bone. Using semiquantitative PCR, we found an increase in OPGL mRNA levels and a decrease in OPG mRNA levels in calvaria of transgenic mice compared with nontransgenic littermate controls. This pattern of OPG and OPGL production would tend to promote osteoclast activation (41) and is similar to the effects of anabolic GPCR agonists in both cell culture systems and in vivo $(35,42,43)$. For example, prostaglandin $\mathrm{E}_{2}$ appears to stimulate OPGL production and inhibits OPG production through adenylyl cyclase-dependent pathways in osteoblast cell lines $(42,53)$. In vivo, PTH potently stimulates OPGL expression and inhibits OPG expression in mice following a single injection of the GPCR agonist (43). Thus, expression of the GRK inhibitor produced a similar effect in transgenic mice without significantly affecting bone histomorphometric measures of osteoclastic activity. The inability of bone histomorphometry to detect enhanced osteoclastic activity may, in part, be related to the sensitivity of the methodology. Alternatively, recent studies suggest that OPGL may be a potent activator of osteoblasts and, when given in pharmacological doses, is a potent bone-forming agent (54). Thus, it is possible that increased OPGL levels affected primarily the osteoblastic component of bone formation and bone remodeling with less pronounced effects on the osteoclasts. Additional studies will be necessary to investigate this latter possibility; however, these data suggest that GRK inhibition in osteoblasts produces a pattern of OPGL and OPG mRNA levels that is seen following pharmacological administration of bone-forming GPCR agonists $(35,42,43)$.

There may be other actions of GRK inhibitors that are mediated downstream of receptor phosphorylation. For example, GPCRs coupled to inhibition of adenylyl cyclase through the $\alpha$-subunit of heterotrimer G proteins also activate phospholipase C (PLC) through $\beta \gamma$ subunit-dependent mechanisms (18). By acting as a $\beta \gamma$ subunit sequestrant, the GRK2-CT inhibits $\beta \gamma$-subunit-dependent activation of PLC (18). These observations may be of importance with regard to the effects of the GRK-CT on bone since, at least with regard to $\mathrm{PTH}$, the anabolic effects of PTH in vivo appear to be mediated by adenylyl cyclase-dependent pathways (55), whereas PLC-dependent pathways either have little effect on bone formation in vivo (55) or may even enhance bone loss in animal model osteoporosis (55). The GRK2-CT may also have effects on additional downstream effectors of GPCR signaling. In this regard, $\beta \gamma$-subunit-dependent pathways stimulate mitogen-activated protein (MAP) kinases in some receptor systems $(56,57)$. By sequestering $\beta \gamma$-subunits, the GRK2-CT has antiproliferative effects in cell culture systems (57). Indeed, because of this antiproliferative effect, we chose to target expression of our transgene to mature osteoblasts, thus avoiding potential antiproliferative effects of GRK inhibition on osteoblast precursor cells (ref. 51 and unpublished data). In future studies, however, it may be of interest to target expression of our transgene to osteoblast precursors using the type 1 collagen promoter or the Cbfa promoter. If the antiproliferative effect of GRK inhibition in cell culture systems also occurs in vivo, it is possible that these transgenic animals will have a reduced number of osteoblasts and might be osteopenic.

In summary, we used the PTH/PTHrP receptor as a model GPCR system to evaluate the effect of GRK inhibition on GPCR responsiveness in bone. We found that expression of a GRK inhibitor enhanced PTH/PTHrP receptor responsiveness both in cells transfected with the $\mathrm{PTH} / \mathrm{PTHrP}$ receptor cDNA as well as in an osteosarcoma cell line that expresses an endogenous receptor for $\mathrm{PTH} / \mathrm{PTH} \mathrm{P}$. Consistent with the apparent attenuation of $\mathrm{PTH} / \mathrm{PTHrP}$ receptor desensitization caused by the GRK inhibitor, enhanced PTH/PTHrP receptor signaling was associated with inhibition of agonist-induced phosphorylation of the PTH/PTHrP receptor. To determine the effect of GRK inhibition on bone formation in vivo, we targeted expression of a GRK inhibitor to mature osteoblasts using the OG2 promoter. Transgenic mice demonstrated an increase in osteoblastic and osteoclastic activity as well as alterations in OPG and OPGL mRNA expression in mouse calvaria, which had a pattern that would tend to promote osteoclast activation. Although both bone formation and bone resorption were enhanced in transgenic mice, the net effect of the transgene was anabolic, indicating that bone formation was increased to a greater extent than bone resorption. Taken together, these data suggest that GRKs play a key role in regulating GPCR systems in bone as well as in modulating bone formation in vivo. The pharmacological manipulation of GRK systems in bone may be a strategy for promoting bone formation in vivo. 


\section{Acknowledgments}

These studies were supported by grants RO1-AR4672 (to R.F. Spurney) and RO1-AR37308 and RO1-AR43468 (to L.D. Quarles) from the NIH National Institute of Arthritis and Musculoskeletal and Skin Diseases.

1. Eastell, R. 1998. Treatment of postmenopausal osteoporosis. N. Engl. J. Med. 338:736-745.

2. Quarles, L.D., and Siddhanti, S.R. 1996. Guanine nucleotide binding-protein coupled signaling pathway regulation of osteoblast-mediated bone formation. J. Bone Min. Res. 11:1375-1383.

3. Quarles, L.D. 1997. Cation sensing receptors in bone: a novel paradigm for regulating bone remodeling? J. Bone Min. Res. 12:1971-1974.

4. Finkelstein, J.S., et al. 1994. Parathyroid hormone for the prevention of bone loss induced by estrogen deficiency. N. Engl. J. Med. 331:1618-1623.

5. High, W.B. 1988. Effects of orally administered prostaglandin $E_{2}$ on cortical bone turnover in adult dogs: a histomorphometric study. Bone. 8:363-373.

6. Liu, C.C., et al. 1991. Preexisting bone loss associated with ovariectomy in rats is reversed by parathyroid hormone. J. Bone Min. Res. 6:1071-1079.

7. Reeve, J., et al. 1980. Anabolic effect of human parathyroid hormone fragment on trabecular bone in involution osteoporosis: a multicentre trial. Br. Med.J. 280:1340-1344.

8. Hock, J.M., and Gera, I. 1992. Effects of continuous and intermittent administration and inhibition of resorption on the anabolic response of bone to parathyroid hormone. J. Bone Min. Res. 7:65-72.

9. Tam, C.S., Heersche, J.N.M., Murray, T.M., and Parsons, J.A. 1982. Parathyroid hormone stimulates the bone apposition rate independently of its resorptive action: differential effects of intermittent and continuous administration. Endocrinology. 100:506-512.

10. Dohlman, H.G., Thorner, J., Caron, M.G., and Lefkowitz, R.J. 1991. Model systems for the study of seven-transmembrane-segment receptors. Annu. Rev. Biochem. 60:653-688.

11. Lefkowitz, R.J. 1993. G-protein-coupled receptor kinases. Cell. 74:409-412.

12. Premont, R.T., Inglese, J., and Lefkowitz, R.J. 1995. Protein kinases that phosphorylate activated $G$ protein-coupled receptors. FASEB J. 9:175-182.

13. Ferguson, S.S.G, Barak, L.S., Zhang, J., and Caron, M.G. 1996. G-proteincoupled receptor regulation: role of G-protein-coupled receptor kinases and arrestins. Can. J. Physiol. Pharmacol. 74:1095-1110.

14. Flannery, P.J., and Spurney, R.F. 2001. Domains of the parathyroid hormone $(\mathrm{PTH})$ receptor required for regulation by $\mathrm{G}$ protein-coupled receptor kinases. Biochem. Pharmacol. 62:1047-1058.

15. Dicker, F., Quitterer, U., Winstel, R., Honold, K., and Lohse, M. 1999. Phosphorylation-independent inhibition of parathyroid hormone receptor signaling by $\mathrm{G}$ protein-coupled receptor kinases. Proc. Natl. Acad. Sci. USA. 96:5476-5481.

16. Malecz, N., Bambino, T., Bencsik, M., and Nissenson, R.A. 1998. Identification of phosphorylation sites in the $\mathrm{G}$ protein-coupled receptor for parathyroid hormone. Receptor phosphorylation is not required for agonist-induced internalization. Mol. Endocrinol. 12:1846-1856.

17. Boekhoff, I., et al. 1994. Olfactory desensitization requires membrane targeting of receptor kinase mediated by $\beta \gamma$-subunits of heterotrimeric $G$ proteins. J. Biol. Chem. 269:37-40.

18. Koch, W.J., Hawes, B.E., Inglese, J., Luttrell, L.M., and Lefkowitz, R.J. 1994 Cellular expression of the carboxyl terminus of a $\mathrm{G}$ protein-coupled receptor kinase attenuates $\mathrm{G}_{\beta \gamma}$-mediated signaling. J. Biol. Chem. 269:6193-6197.

19. Koch, W.J., et al. 1995. Cardiac function in mice overexpressing the $\beta$-adrenergic receptor kinase or a $\beta$ ARK inhibitor. Science. 268:1350-1353.

20. Koch, W.J., Inglese, J., Stone, C., and Lefkowitz, R.J. 1993. The binding site for the $\beta \gamma$ subunits of heterotrimeric $G$ proteins on the $\beta$-adrenergic receptor kinase. J. Biol. Chem. 268:8256-8260.

21. Corral, D.A., et al. 1998. Dissociation between bone resorption and bone formation in osteopenic transgenic mice. Proc. Natl. Acad. Sci. USA 95:13835-13840

22. Aubin, J.E. 1998. Advances in the osteoblast lineage. Biochem. Cell Biol. 76:899-910.

23. Liu, S., Guo, R., and Quarles, L.D. 2001. Cloning and characterization of the proximal murine Phex promoter. Endocrinology. 142:3987-3995.

24. McCowage, G.B., et al. 1998. Multiparameter-fluorescence activated cell sorting analysis of retroviral vector gene transfer into primitive umbilical cord blood cells. Exp. Hematol. 26:288-298.

25. Arriza, J.L., et al. 1992. The G-protein-coupled receptor kinases BARK1 and BARK2 are widely distributed at synapses in rat brain. J. Neurosci. 12:4045-4055.

26. Abou-Samra, A.B., et al. 1992. Expression cloning of a common receptor for parathyroid hormone and parathyroid hormone-related peptide from rat osteoblast-like cells: a single receptor stimulates intracellular accumulation of both cAMP and inositol trisphosphates and increases intracellular free calcium. Proc. Natl. Acad. Sci. USA. 89:2732-2736.
27. Howry, R.P., et al. 2000. An in vitro system for efficiently evaluating gene therapy approaches to hemoglobinopathies. Gene Ther. 7:215-223.

28. Sambrook, J., Fritch, E.F., and Maniatis, T. 1989. Molecular cloning: a labora tory manual. N. Ford, editor. Cold Spring Harbor Laboratory Press. Cold Spring Harbor, New York, USA.

29. Scatchard, G. 1949. Attraction of proteins for small molecules and ions. Ann. NY Acad. Sci. 51:660-672.

30. Salomon, Y., Londos, C., and Rodbell, M. 1974. A highly sensitive adenylate cyclase assay. Anal. Biochem. 58:541-548.

31. Laemmli, U.K. 1970. Cleavage of structural proteins during the assembly of the head of bacteriophage T4. Nature. 227:680-685.

32. Marotta, C.A., Wilson, J.T., Forget, B.G., and Weissman, S.M. 1977. Human $\beta$-globulin messenger RNA. J. Biol. Chem. 252:5040-5053.

33. Benovic, J.L., DeBasi, A., Stone, W.C., Caron, M.G., and Lefkowitz, R.J. 1989 $\beta$-adrenergic kinase: primary structure delineates a multigene family. Science. 246:235-240

34. Takai, H., et al. 1998. Transforming growth factor- $\beta$ stimulates the production of osteoprotegerin/osteoclastogenesis inhibitory factor by bone marrow stromal cells. J. Biol. Chem. 273:27091-27096.

35. Lee, S.K., and Lorenzo, J.A. 1999. Parathyroid hormone stimulates TRANCE and inhibits osteoprotegerin messenger ribonucleic acid expression in murine bone marrow cultures: correlation with osteoclast-like cell formation. Endocrinology. 140:3552-3561.

36. Garner, S.C., Pi, M., Tu, Q., and Quarles, L.D. 2001. Rickets in cationsensing receptor-deficient mice: an unexpected skeletal phenotype. Endocrinology. 142:3996-4005.

37. Parfitt, A.M., et al. 1987. Bone histomorphometry: standardization of nomenclature, symbols and units. J. Bone Min. Res. 2:595-610.

38. Fukayama, S., Kong, G., Benovic, L., Meurer, E., and Tashjian, A.H. 1997. $\beta$-adrenergic receptor kinase-1 acutely regulates $\mathrm{PTH} / \mathrm{PTHrP}$ receptor signaling in human osteoblast like cells. Cell. Signal. 9:467-474.

39. Frendo, J.L., et al. 1998. Functional hierarchy between two OSE2 elements in the control of osteocalcin gene expression in vivo. J. Biol. Chem. 273:30509-30516.

40. Jilka, R.L., et al. 1998. Loss of estrogen upregulates osteoblastogenesis in the murine bone marrow. Evidence for autonomy from factors released during bone resorption. J. Clin. Invest. 101:1942-1950.

41. Takahashi, N., Udagawa, N., and Suda, T. 1999. A new member of the tumor necrosis factor ligand family, ODF/OPGL/TRANCE/RANKL, regulates osteoclast differentiation and function. Biochem. Biophys. Res. Commun. 256:229-255.

42. Brandstrom, H., et al. 1998. Regulation of osteoprotegerin mRNA levels by prostaglandin $\mathrm{E}_{2}$ in human bone marrow stromal cells. Biochem. Biophys. Res. Commun. 247:338-341.

43. Onyia, J.E., et al. 2000. In vivo demonstration that human parathyroid hormone 1-38 inhibits the expression of osteoprotegerin in bone with the kinetics of an immediate early gene. J. Bone Min. Res. 15:863-871.

44. Mundy, G.R. 1996. Bone-resorbing cells. In Primer of metabolic bone diseases and disorders of mineral metabolism. M.J. Favus, editor. Lippincott-Raven Publishers. New York, New York, USA. 16-24.

45. Puzas, J.E. 1996. Osteoblast cell biology: lineage and functions. In Primer of metabolic bone diseases and disorders of mineral metabolism. M.J. Favus, editor. Lippincott-Raven Publishers. New York, New York, USA. 1-16.

46. Strewler, G.L. 2000. The physiology of parathyroid hormone-related protein. N. Engl.J. Med.342:177-185.

47. Lanske, B., et al. 1996. PTH/PTHrP receptor in early development and Indian hedgehog-regulated bone growth. Science. 273:663-666.

48. Saino, H., Matsuyama, T., Takada, J., Kaku, T., and Ishii, S. 1997. Long-term treatment of indomethacin reduces vertebral bone mass and strength in ovariectomized rats. J. Bone Miner. Res. 12:1844-1850.

49. Okada, Y., et al. 1999. Effects of prostaglandin G/H synthase-2 knockout on bone. J. Bone Miner. Res. 14(Suppl. 1):173. (Abstr.)

50. Susa, M. 1999. Heterotrimeric $\mathrm{G}$ proteins as fluoride targets in bone. Int. J. Mol. Med. 3:115-126

51. Bliziotes, M., Gunness, M., Zhang, X.W., Nissenson, R., and Wiren, K. 2000 Reduced G-protein-coupled-receptor kinase 2 activity results in impairment of osteoblast function. Bone. 27:367-373.

52. Jilka, R.J., et al. 1999. Increased bone formation by prevention of osteoblast apoptosis with parathyroid hormone. J. Clin. Invest. 104:439-446.

53. Li, W., et al. 2000. Knockout of the murine prostaglandin EP2 receptor impairs osteoclastogenesis in vitro. Endocrinology. 141:2054-2061.

54. Lam, J., Ross, R.P., and Teitelbaum, L. 2001. RANK ligand stimulates anabolic bone formation. J. Bone Miner. Res. 16(Suppl. 1):150. (Abstr.)

55. Armamento-Villareal, R., et al. 1997. An intact $\mathrm{N}$ terminus is required for the anabolic action of parathyroid hormone on adult female rats. J. Bone Miner. Res. 12:384-392.

56. Daaka, Y., Luttrell, L.M., and Lefkowitz, R.J. 1997. Switching of the coupling of the $\beta_{2}$ adrenergic receptor to different $G$ proteins by protein kinase $A$ Nature. 390:88-91.

57. Van Bieson, T., Luttrell, L.M., Hawes, B.E., and Lefkowitz, R.J. 1996. Mitogenic signaling via $G$ protein coupled receptors. Endocr. Rev. 17:698-714. 